\title{
Article
}

Subscriber access provided by King Abdullah University of Science and Technology Library

\section{Charge Carrier Generation Followed by Triplet State Formation, Annihilation, and Carrier Recreation in PBDTTT-C:PCBM Photovoltaic Blends}

\author{
Dominik W. Gehrig, lan A. Howard, and Frédéric Laquai
}

J. Phys. Chem. C, Just Accepted Manuscript • DOI: 10.1021/acs.jpcc.5b03467 • Publication Date (Web): 22 May 2015

Downloaded from http://pubs.acs.org on May 25, 2015

\section{Just Accepted}

"Just Accepted" manuscripts have been peer-reviewed and accepted for publication. They are posted online prior to technical editing, formatting for publication and author proofing. The American Chemical Society provides "Just Accepted" as a free service to the research community to expedite the dissemination of scientific material as soon as possible after acceptance. "Just Accepted" manuscripts appear in full in PDF format accompanied by an HTML abstract. "Just Accepted" manuscripts have been fully peer reviewed, but should not be considered the official version of record. They are accessible to all readers and citable by the Digital Object Identifier (DOI®). "Just Accepted" is an optional service offered to authors. Therefore, the "Just Accepted" Web site may not include all articles that will be published in the journal. After a manuscript is technically edited and formatted, it will be removed from the "Just Accepted" Web site and published as an ASAP article. Note that technical editing may introduce minor changes to the manuscript text and/or graphics which could affect content, and all legal disclaimers and ethical guidelines that apply to the journal pertain. ACS cannot be held responsible for errors or consequences arising from the use of information contained in these "Just Accepted" manuscripts. 


\title{
Charge Carrier Generation Followed by Triplet State
}

\section{Formation, Annihilation, and Carrier Recreation in}

\section{PBDTTT-C:PC ${ }_{60}$ BM Photovoltaic Blends}

\author{
Dominik. W. Gehrig, Ian A. Howard, Frédéric Laquai*† \\ Max Planck Research Group for Organic Optoelectronics, Max Planck Institute for Polymer \\ Research, Ackermannweg 10, 55128 Mainz, Germany
}

\section{AUTHOR INFORMATION \\ Corresponding Author \\ *E-mail: laquai@mpip-mainz.mpg.de}

\author{
Present Addresses \\ $†$ Physical Sciences and Engineering Division (PSE), Material Science and Engineering (MSE), \\ Solar and Photovoltaics Engineering Research Center (SPERC), King Abdullah University of \\ Science and Technology (KAUST), Thuwal 23955-6900, Kingdom of Saudi Arabia. E-mail: \\ frederic.laquai@kaust.edu.sa
}


Triplet state formation after photoexcitation of low-bandgap polymer:fullerene blends has recently been demonstrated, however, the precise mechanism and its impact on solar cell performance is still under debate. Here, we study exciton dissociation, charge carrier generation and triplet state formation in low-bandgap polymer PBDTTT-C:PC ${ }_{60} \mathrm{BM}$ bulk heterojunction photovoltaic blends by a combination of fs- $\mu$ s broadband Vis-NIR transient absorption (TA) pump-probe spectroscopy and multivariate curve resolution (MCR) data analysis. We found subps exciton dissociation and charge generation followed by sub-ns triplet state creation. The carrier dynamics and triplet state dynamics exhibited a very pronounced intensity dependence indicating non-geminate recombination of free carriers is the origin of triplet formation in these blends. Triplets were found to be the dominant state present on the nanosecond timescale. Surprisingly, the carrier population increased again on the ns- $\mu$ s timescale. We attribute this to triplet-triplet annihilation and the formation of higher energy excited states that subsequently underwent charge transfer. This unique dip and recovery of the charge population is a clear indication that triplets are formed by non-geminate recombination, as such a kinetic is incompatible with a monomolecular triplet state formation process.

KEYWORDS charge generation, low-bandgap polymers, multivariate curve resolution, organic solar cells, transient absorption spectroscopy, triplet states 


\section{INTRODUCTION}

Triplet state formation is a ubiquitous phenomenon in organic semiconductors and typically occurs as a consequence of intersystem crossing from the singlet excited state or via fission of high energy excitations. ${ }^{1-2}$ It plays a prominent role in the device physics of organic lightemitting diodes (OLEDs), in which recombination of injected charge carriers should theoretically lead to a fraction of $75 \%$ triplet state formation according to spin statistics. Recently, increasing attention is being paid to triplet states in polymer:fullerene photovoltaic blends. ${ }^{3-5}$ In the last year, several groups have reported triplet state formation in low-bandgap polymer:fullerene solar cells, but the precise mechanism behind the triplet state formation is currently debated. An understanding of whether triplets are formed due to geminate recombination of interfacial charge-transfer (CT) states created upon exciton dissociation or due to non-geminate recombination of spatially-separated charges (SSC) is still sought. It is important to know the exact nature of the triplet state formation mechanism because a geminate recombination mechanism would be an intensity-independent process and thus play a significant role even under solar illumination conditions (that is at lower carrier concentrations than transient absorption experiments). On the other hand, non-geminate recombination rates depend strongly on the charge carrier concentrations and therefore may be of more relevance after pulsed laser excitation, and less relevant for photovoltaic devices performance under standard illumination. In the past Westenhoff et al. studied all-polymer photovoltaic blends by transient absorption spectroscopy and reported triplet state formation as a consequence of recombination of interfacial charge-transfer states. ${ }^{6}$ Di Nuzzo et al. investigated triplet state formation in PCPDTBT:PCBM blends and its dependence on film morphology by steady-state photoinduced absorption spectroscopy. The authors argued that processing of the blend in the presence of 
solvent additives changes the blend morphology and energy landscape and thereby reduces triplet state formation as the triplet level becomes energetically less accessible in blends prepared with additives. ${ }^{7}$ However, a recent transient absorption study on the same material system by Chow et al. presented contradictory evidence. They showed that in fact more triplets are created in the blend processed with solvent additives, and that this is a consequence of an increased yield of free charge carriers that create triplets through non-geminate recombination. ${ }^{5}$ Similarly, Rao et al. showed that triplet formation in PCPDTBT:PCBM blends is depending on the excitation density and concluded that triplet states are populated via bimolecular recombination events. ${ }^{3}$ Very recently, Dimitrov et al. reported polaron pair-mediated triplet state formation caused by recombination of interfacial states created upon singlet exciton dissociation in a fluorinated lowbandgap polymer:fullerene blend. ${ }^{4}$ Furthermore, triplet state formation via non-geminate recombination of free charges has very recently been identified as a loss channel in PTB7:PC ${ }_{60} \mathrm{BM}$ solar cells. ${ }^{8}$ Interestingly, the triplet state population could be suppressed by addition of a $1 / 2$ spin-radical, namely galvinoxyl, in turn leading to a significant improvement of the device photocurrent. In our own work we very recently compared triplet state formation in the silicon-substituted analogue of PCPDTBT, namely PSBTBT, and showed that triplet states are created on a sub-ns timescale in PCPDTBT:PCBM supporting the findings of Chow et al. We found that triplets were also created in PSBTBT:PCBM, however, to a much lesser extent than in the PCPDTBT:PCBM which indicated a suppressed rate of non-geminate recombination contributes to PSBTBT:PCBM's higher photovoltaic efficiency. ${ }^{9}$ 


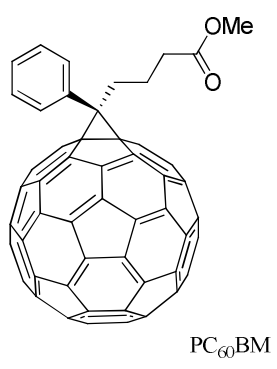

Scheme 1. Chemical structures of the material system investigated in the present study, namely the low-bandgap donor polymer PBDTTT-C and the electron acceptor $\mathrm{PC}_{60} \mathrm{BM}$.

In the present study we investigated the excited state dynamics, specifically the charge recombination and triplet state formation, following pulsed laser excitation of another widely used prototypic low-bandgap polymer:fullerene blend, namely PBDTTT-C:PC ${ }_{60} \mathrm{BM}$ (see Scheme 1 for chemical structures), by ps- $\mu$ s broadband Vis-NIR transient absorption spectroscopy in combination with multivariate curve resolution (MCR) analysis of the experimental data. The BDT comonomer is a common structural motif often used in alternating donor-acceptor low-bandgap polymers, thus the investigated PBDTTT-C polymer belongs to a whole family of structurally-related materials very often used in polymer solar cells such as PTB1 and PTB7 for instance. Compared to the PBDTTT-C polymer studied by us, in which the thienothiophene (TT) comonomer is substituted with an ethyl-hexyloxy side group and the BDT unit is substituted with an 2-ethyl-hexan-1-one side group, PTB1 is substituted with a dodecylester group at the TT unit and n-octyloxy side chains at the BDT comonomer, while in PTB7 the BDT unit is the same as in PBDTTT-C and the TT unit is substituted with an ethyl-hexyl ester and in addition also fluorinated in comparison to PTB1 and PBDTTT-C. ${ }^{10-12}$ Guo et al. and Rolczynski et al. have previously studied the correlation between bulk morphology, excited state dynamics and power conversion efficiency of bulk heterojunction solar cells using structurally- 
related donor polymers including PTB1, ${ }^{13-15}$ while Carsten et al. and Szarko et al. have studied the photophysics of PTB7 blended with fullerenes. ${ }^{16-18}$ However, in the aforementioned studies the authors limited their spectroscopic experiments to the ps-ns timescale mostly relevant for exciton dissociation and charge carrier formation, while the ns- $\mu$ s timerange relevant for charge carrier recombination was not investigated, and furthermore none of the studies reported triplet state formation in blends of these polymers with fullerene derivatives. Here, we use a combination of broadband Vis-NIR transient absorption spectroscopy across a dynamic range from femto- to milliseconds in conjunction with sophisticated MCR analysis to separate the individual components contributing to the experimentally-observed data and thereby to determine the component-associated spectra and their dynamics. Specifically, we demonstrate in the following that in the investigated PBDTTT-C:PCBM blends triplet states are rapidly generated by non-geminate recombination on a sub-ns timescale and subsequently undergo fast triplet-triplet annihilation on the ns- $\mu$ s timescale that leads to a recreation of the charge carrier population. 


\section{RESULTS \& DISCUSSION}
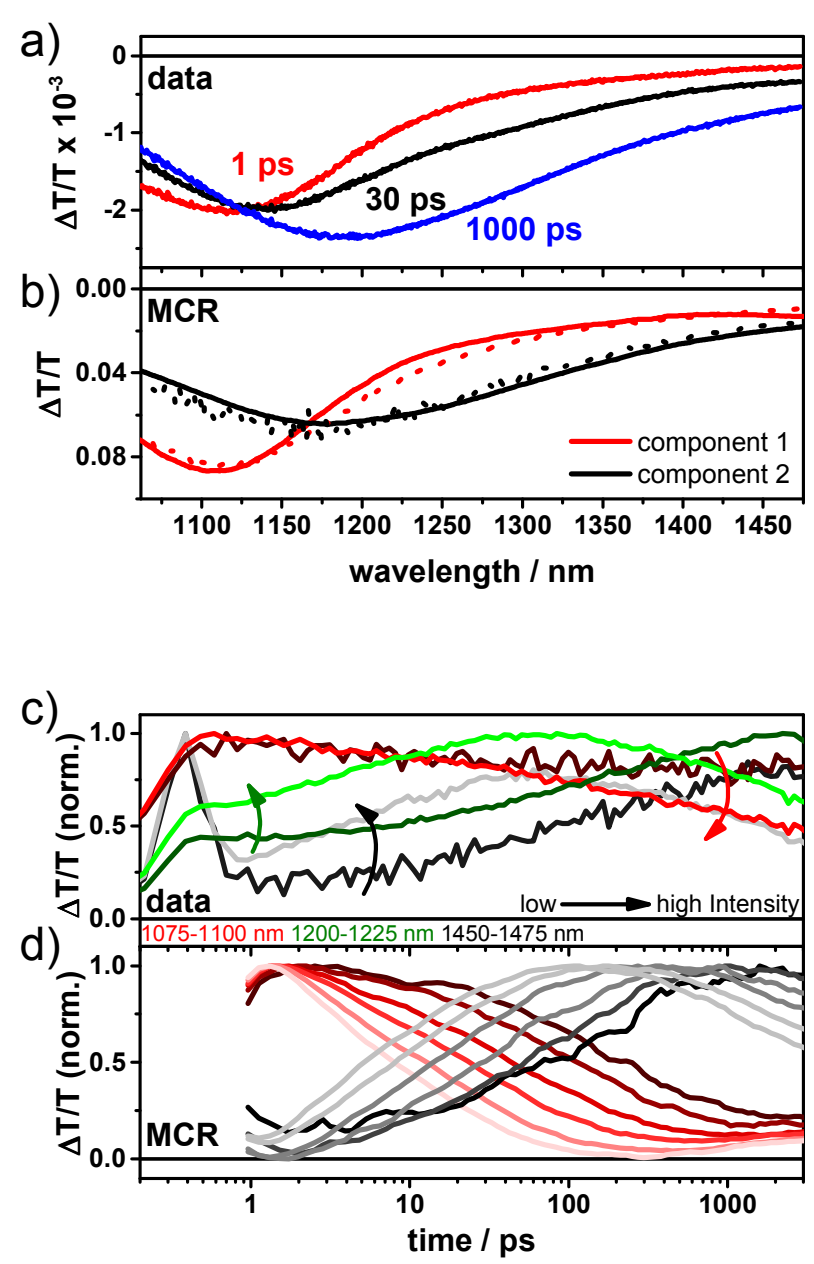

Figure 1. a) ps-ns transient absorption spectra at different delay times after excitation of the polymer at $650 \mathrm{~nm}$ with a fluence of $6.7 \mu \mathrm{J} / \mathrm{cm}^{2}$. b) Component spectra obtained by MCR-ALS analysis of the ps-ns TA data using non-negativity as a constraint for the concentration profiles and non-positivity as spectral constraint. The dotted spectra represent the charge- and tripletinduced absorption spectra obtained by separate measurements on a polymer:PDI blend and a triplet sensitizer-doped polymer film, respectively. c) Dynamics extracted at 1075-1100 nm (red), 1200-1225 nm (green), and 1450-1475 nm (grey) at different excitation densities. d) Dynamics of component 1 (charges) and 2 (triplets) obtained by MCR-ALS analysis of the TA data after 1 ps. Note the pronounced intensity dependence of the dynamics. 
Figure 1 a) shows the near-infrared ps-ns transient absorption spectra of a PBDTTT-C:PCBM (1:2) bulk heterojunction blend film as typically used in photovoltaic devices and c) the signal dynamics monitored in selected wavelength regions, which, as we will show, correspond to specific excited states (thereafter named components) present in the blend film after photoexcitation. The sub-ps TA spectrum (shown in the SI) exhibits contributions of the excited state absorption of the primary photoexcitations, namely singlet exciton-induced absorption $\left(\mathrm{PA}_{\mathrm{Ex}}\right)$. Polymer singlet excitons undergo ultrafast charge transfer in the blend as indicated by the rapid decay of the $\mathrm{PA}_{\mathrm{Ex}}$ at around $1450-1475 \mathrm{~nm}$, that is a wavelength range, in which singlet excitons clearly dominate the TA spectra at early delay times. For comparison we have included a TA spectrum of a pristine polymer film in the SI. The TA spectrum 1 ps after photoexcitation peaks at $1120 \mathrm{~nm}$, but rapidly undergoes a spectral evolution on the sub-ns timescale. In fact, a progressive red-shift of the maximum of the TA signal with time was observed until at about $1 \mathrm{~ns}$ the photoinduced absorption peaked at $1190 \mathrm{~nm}$. Interestingly, we also observed an isosbestic point at $1135 \mathrm{~nm}$ indicating that we witnessed a transition between two components without any significant loss of the total excited state population by recombination of the excited states to the ground state. In conjunction with the red-shift of the photoinduced absorption we also observed an increase in the total signal amplitude indicating that the product created on the sub-ns timescale, hereafter referred to as component 2, has a larger excited state absorption cross section than component 1 , that is the product of exciton dissociation obtained on the ps timescale. In order to gain more detailed insight into the nature of the components we performed multivariate curve resolution analysis on the experimentally-measured TA data, a soft-modelling data analysis technique previously introduced by Jaumot et al. ${ }^{19}$ We have recently reviewed this technique and its usefulness for the analysis of TA data and we have previously applied it for TA 
data analysis of different photovoltaic systems, for instance polymer:perylene diimide (PDI) blends, small molecule donor:PDI acceptor systems and low-bandgap polymer:fullerene blends. ${ }^{9}$, 20-22 A brief introduction to the principles of MCR analysis is also presented in the Supporting Information. The MCR analysis of the experimental TA data yielded two separate component spectra and component-associated dynamics as shown in Figure $1 \mathrm{~b}$ ) and d), respectively.

However, the precise nature of the excited states is a priori unknown and thus we performed control experiments to identify the two components. In fact, component 1 , which is already present at 1 ps after photoexcitation when polymer exciton dissociation is completed and which peaks at $1120 \mathrm{~nm}$ could be assigned to charges, as the peak position of the component-associated spectrum matches very closely that of the charge-induced absorption ( $\left.\mathrm{PA}_{\text {Charge }}\right)$ obtained on an iodine-oxidized polymer film (see SI) and also previously reported for the same polymer but blended with PDI as acceptor instead of fullerene (see Figure 1). ${ }^{21}$ We note that this component spectrum is also similar in shape and position as the charge-induced absorption spectrum reported earlier by Guo et al. for the structurally-related polymer PTB1 when blended with PCBM. ${ }^{13}$ The spectrum of component 2 was found to be virtually the same as the triplet-induced absorption ( $\left.\mathrm{PA}_{\text {Triplet }}\right)$ obtained by TA experiments on polymer films doped with different metallated porphyrins as sensitizers (see SI and figure 1). Hence, we can confidently conclude that component 1 represents charges, while component 2 triplet states in the PBDTTT$\mathrm{C}: \mathrm{PC}_{60} \mathrm{BM}$ blend. Furthermore, the excellent agreement of the spectra obtained from the MCR with the known spectra means that the factorization provided by the MCR is accurate, and the dynamics it extracted accurately correspond to the real evolution of the charge and triplet concentrations. The component-associated dynamics from the MCR analysis are shown in figure $1 \mathrm{~d})$. Both the charge carrier dynamics as well as the triplet state dynamics exhibit a very 
pronounced dependence on the excitation fluence on the sub-ns timescale, that is, on the total concentration of excited states. The decrease in the charge population occurs more quickly at higher fluences, in line with the observation that the increase in the triplet population also occurs more quickly at higher fluences. The timescale of the charge decay and triplet formation are clearly linked, and change similarly as a function of fluence. These observations clearly point to a mechanism of triplet state formation via non-geminate recombination of charges, in line with previous reports for TQ1:PCBM by Rao et al., PCPDTBT:PCBM by Chow et al. and PSBTBT:PCBM by Etzold et al. ${ }^{3,5,9}$ In order to estimate the sub-ns recombination coefficient we parameterized the intensity-dependent dynamics of component 1 by a combination of a concomitant single exponential and non-geminate (power law) process. The former accounts for any fast recombination and annihilation process of charges, while the latter describes the carrier population undergoing sub-ns non-geminate recombination. Interestingly, this simplified approximation yields a non-geminate recombination coefficient on the order of $10^{-9} \mathrm{~cm}^{3} \mathrm{~s}^{-1}$ which translates into an effective bimolecular recombination coefficient at a carrier concentration of $5 \times 10^{15} \mathrm{~cm}^{-3}$ (approximately that under solar illumination) on the order of $10^{-10} \mathrm{~cm}^{3} \mathrm{~s}^{-1}$. This is very high and in line with the fast sub-ns charge recombination observed experimentally. Using the latter value to calculate the minimum mobility of electrons and holes according to the approach previously reported by Koster et al. ${ }^{23}$ yields a mobility value of $\sim 1 \mathrm{~cm}^{2}(\mathrm{Vs})^{-1}$. This rather high mobility is in good agreement with values used in Kinetic Monte Carlo simulations by Burke et al. to explain the high exciton dissociation yield in bulk heterojunction solar cells and it is also compatible with conductivity values derived from ultrafast terahertz spectroscopy experiments on polymer:fullerene blends. ${ }^{24-25}$ This is also consistent with the picture that charges created by ultrafast exciton dissociation are located in the upper part of the density of states 
(DOS) and thus are very mobile as they have not yet relaxed within the density of states to sites of lower energy in the tail of the DOS.

Furthermore, we determined the TA signal amplitude of charges and triplets from the MCR analysis and plotted the maximum TA signal versus the excitation energy or pump fluence (see SI). Interestingly, the maximum charge carrier and triplet concentration exhibit a similar and linear dependence on the excitation fluence. For the charge carriers this is obvious, as the initial carrier concentration at $1 \mathrm{ps}$ should be directly proportional to the fluence as long as sub-ps higher order annihilation processes do not play a role and the ground state absorption is not saturated. However, the reason for the linear increase of the triplet yield with fluence is not immediately apparent, but it does not necessarily indicate that the triplets are created from a monomolecular process such as spin-flipping in a CT state. Rather, charge recombination is clearly dominated by non-geminate recombination in the range of fluences we investigated, as indicated by the intensity dependent dynamics. This supports that for all of these fluences or charge carrier densities created by pulsed laser excitation essentially all generated charges undergo non-geminate recombination and thereby cause the triplet yield of this bimolecular process nonetheless to be linearly dependent on the initial charge carrier concentration. In this case the rate of triplet formation should depend on fluence and in fact, we observed a clear correlation between the rate of non-geminate recombination and triplet formation across the measured fluences as obvious from Figure $1 \mathrm{~d}$ ). Furthermore, we note that, although most of the initially-created charges had recombined by $1 \mathrm{~ns}$, the decay dynamics on the ns- $\mu$ s timescale were still intensity-dependent indicating, as we will discuss later, that triplet-triplet annihilation is a major recombination mechanism for triplets at these fluences. Nevertheless, we still obtained a reasonable photovoltaic performance (see SI) from the PBDTTT-C:PC ${ }_{60} \mathrm{BM}$ blends, which 
would not be the case, if the same processes occurred to a similar extent under solar illumination, that is, at much lower charge carrier densities. Hence, our results clearly point to a non-geminate mechanism as the origin of triplet state formation in this particular material system.
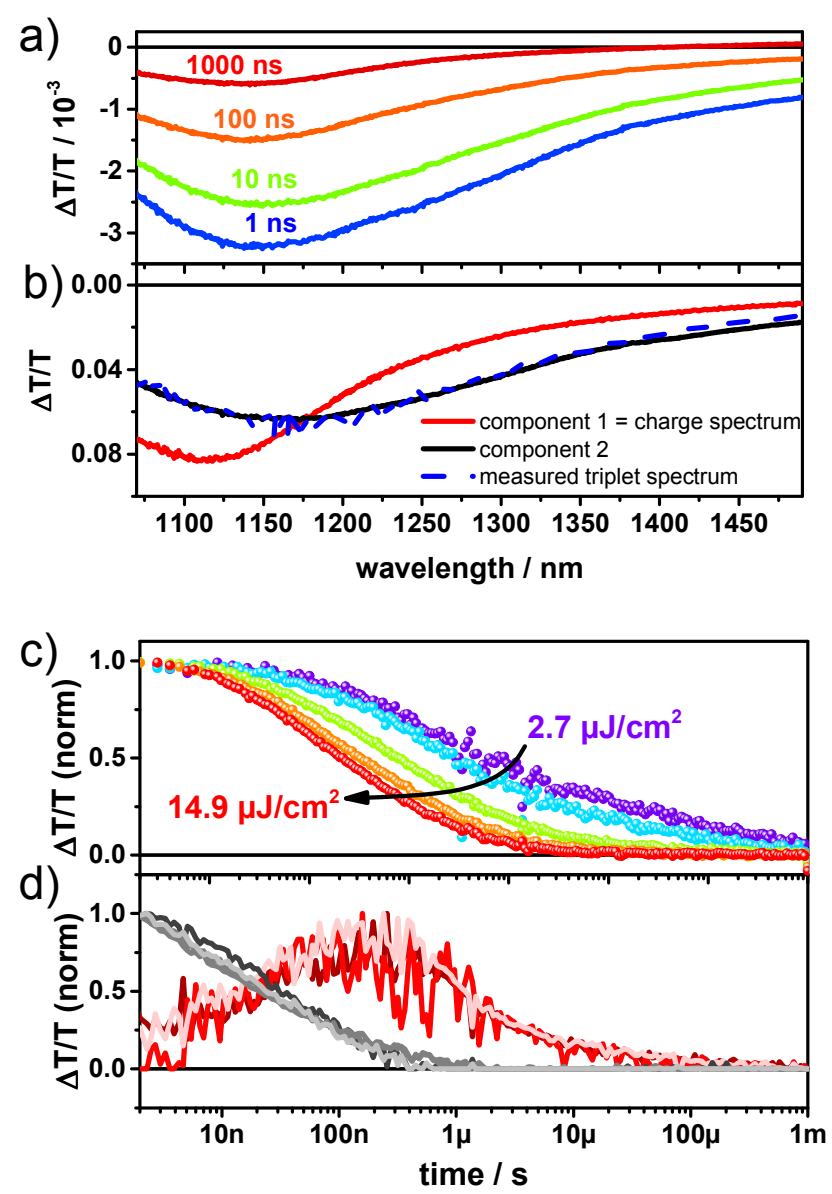

Figure 2. a) ns- $\mu$ s TA spectra in the near-infrared spectral region measured after pulsed laser excitation at $532 \mathrm{~nm}$ and $8.5 \mu \mathrm{J} / \mathrm{cm}^{2}$ per pulse. b) Absorption spectra extracted from the $\mathrm{ns}-\mu \mathrm{s}$ data surface by MCR-ALS analysis. The black spectrum represents component 2 obtained by using the charge-induced absorption spectrum of a PBDTTT-C:PDI blend as input, while the dashed blue spectrum corresponds to the triplet-induced absorption spectrum obtained on a PtOEP-sensitized film. c) Dynamics observed at different excitation densities extracted in the 
spectral region from 1140 to $1160 \mathrm{~nm}$. d) Intensity dependence of the concentration profiles for component 1 (red, charges) and component 2 (grey, triplets).

Figure 2 a) shows the experimentally determined ns- $\mu$ s TA spectra after pulsed laser excitation of the same blend as used for the ps-ns experiments. The signal dynamics tracked between 1140-1160 nm are clearly intensity-dependent as shown in Figure 2 c). Similar to the analysis of the ps-ns dynamics we also performed MCR-ALS analysis of the TA data obtained on the ns- $\mu \mathrm{s}$ timescale. Again, we observed two components are required to describe the entire TA data matrix, of which component 1 represents the charge-induced absorption very similar to the charge-induced absorption spectrum of a PBDTTT-C:PDI blend. Component 2 represents the triplet-induced absorption, as supported by comparison of this component spectrum with the separately obtained absorption spectrum of triplet states also depicted in Figure 2 b). We observed that the triplet decay dynamics are only weakly intensity-dependent as shown in Figure 2 d) and exhibit a rather complex decay pattern, which can neither be described by an exponential decay nor a power law indicating that several parallel processes determine the triplet decay including the recombination of triplets to the ground state, as well as triplet-triplet and perhaps triplet-charge annihilation. In fact, the observed triplet state decay dynamics are similar to those observed in the porphyrin-doped polymer film at high excitation densities, that is, under conditions which lead to a significant population of polymer triplet states and thus cause triplettriplet annihilation to be the main decay channel of the triplet state population.

Surprisingly, we also observed a pronounced rise of the charge-induced absorption up to $\sim 200 \mathrm{~ns}$ (see figure $2 \mathrm{~d}$ ), which is the time scale on which the main part of the triplet population decays. This is rather unexpected and indicates that a substantial fraction of the triplet population indeed underwent triplet-triplet annihilation, thereby created higher energy triplet and singlet excitons, 
which in turn had sufficient energy to undergo charge separation and led to a delayed (re)generation of charge carriers in the polymer. As these regenerated charges create a charge density that is much lower than the original charge density immediately after optical excitation, the regenerated charges live much longer and are not immediately lost to non-geminate recombination. This longer lifetime of the regenerated charges allows them to be clearly observed. The triplet-triplet annihilation is supported by the fast decay of the triplet states observed in the polymer:fullerene blend which is similar to the high excitation conditions in the triplet sensitizer-doped film as outlined above.
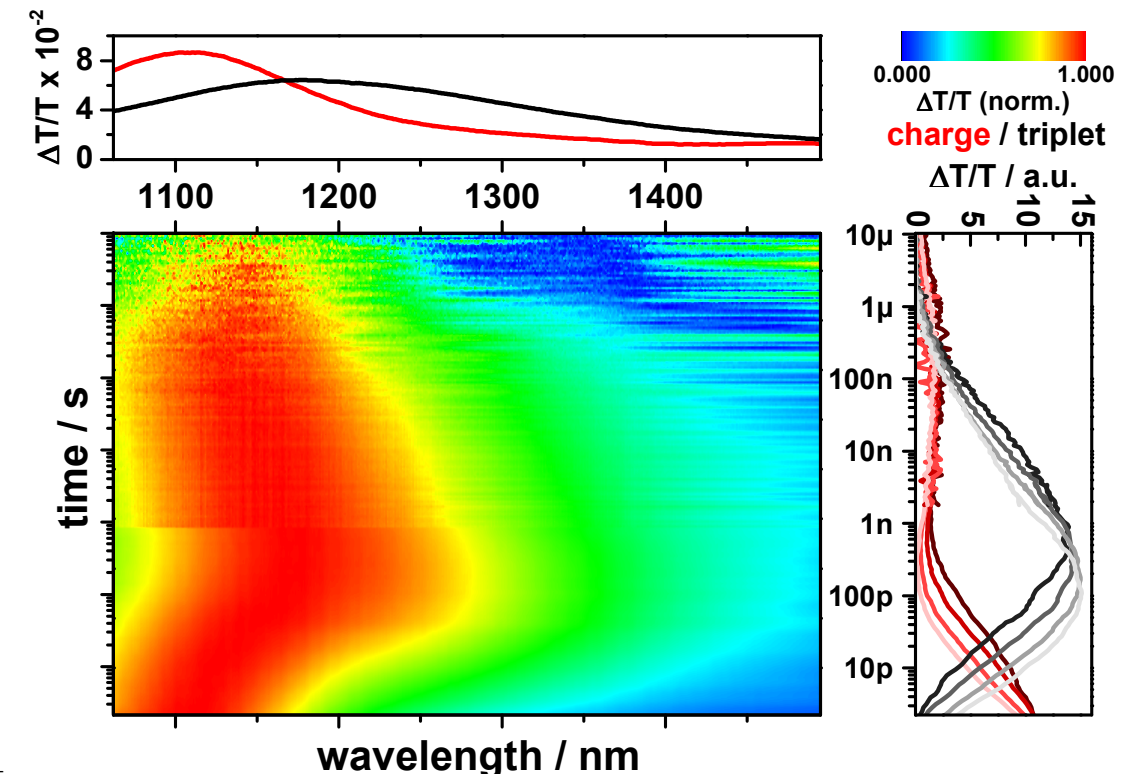
Figure 3 shows a combined contour plot of the ps- $\mu$ s experimental TA data and the corresponding charge-induced and triplet-induced absorption spectra and dynamics as obtained by MCR analysis of the ps-ns TA data and ns- $\mu$ s TA data. Clearly, the charge carrier concentration is minimal at around $1 \mathrm{~ns}$ after excitation. This depletion of the pool of charge carriers in conjunction with the isosbestic point observed in the ps-ns TA data points to a very high triplet state yield, likely close to being quantitative under the experimental conditions. Furthermore, the combination of the TA data across the entire ps- $\mu$ s timerange allows us to estimate that the charge carrier regeneration on the ns- $\mu$ s timescale leads to not more than a quarter of the initial carrier population that was initially present after photoexcitation. In principle, if all processes, that is non-geminate recombination, triplet formation, triplet-triplet annihilation and carrier regeneration were quantitative, the delayed carrier population could reach about half of the initial value as the recreation of one charge carrier requires the annihilation of two triplet states. Taking into account the close to quantitative triplet state formation process on the ns timescale, this observation, precisely that only about a quarter of the initial carrier population is regenerated, points to the presence of additional loss channels, which likely occur during the triplet-triplet annihilation and charge carrier recreation process.

\section{CONCLUSIONS}

In summary, we have demonstrated by a combination of Vis-NIR transient absorption spectroscopy over nine orders of magnitude in time and multivariate curve resolution analysis of the experimental TA data that after photoexcitation of PBDTTT-C:PC ${ }_{60} \mathrm{BM}$ blends by pulsed laser excitation a cascade of photophysical processes is initiated starting with ultrafast exciton dissociation, followed by a fast sub-ns decay of the charge carrier population and almost quantitative formation of triplet states. Further analysis of the carrier and triplet state dynamics 
revealed a pronounced intensity dependence of the dynamics pointing to a non-geminate recombination of charges and origin of triplet state formation. A non-geminate recombination coefficient around $10^{-10} \mathrm{~cm}^{3} \mathrm{~s}^{-1}$ was determined for the sub-ns charge recombination pointing to an initial mobility of carriers as high as $1 \mathrm{~cm}^{2}(\mathrm{Vs})^{-1}$. The triplet state population is followed by triplet-triplet-annihilation on the ns- $\mu$ s timescale, while in parallel a significant charge carrier population is recreated. We believe that the charges are recreated by dissociation of high energy singlet and triplet states created by triplet-triplet-annihilation. Despite the close to quantitative triplet formation on the sub-ns timescale following pulsed laser excitation we still obtained a moderate photovoltaic performance from this blend under solar illumination indicating that the yield of triplets must be significantly reduced at lower photon flux. Overall, our results provide insight into the ongoing debate of the mechanism of triplet state formation in low-bandgap polymer:fullerene blends, the fate of triplet states after their generation and implications for the photovoltaic device performance and future material development. 


\section{EXPERIMENTAL METHODS}

Thin film preparation. PBDTTT-C $\left(\mathrm{Mn}=132.000 \mathrm{~kg} \mathrm{~mol}^{-1}\right)$ and $\mathrm{PC}_{60} \mathrm{BM}$ were dissolved separately in chlorobenzene at a concentration of $15 \mathrm{mg} \mathrm{mL}^{-1}$ and stirred at $70{ }^{\circ} \mathrm{C}$ overnight. The solutions were mixed at a 1:2 ratio $2 \mathrm{~h}$ prior to spin coating at a rotation speed of $1000 \mathrm{rpm}$. For spectroscopic samples the active layer was spincoated on quartz substrates which were subsequently cleaned by ultrasonication in detergent, acetone and iso-propanol. Subsequently, the samples were treated with an argon plasma for $15 \mathrm{~min}$. For photovoltaic devices, a $\sim 40 \mathrm{~nm}$ thick poly(3,4-ehylene-dioxythiophene):poly(styrenesulfonate) (PEDOT:PSS) (Clevios P VP Al 4083, H.C. Stark) was spincoated on ITO-coated glass substrates (Präzisions Glas \& Optic $\mathrm{GmbH}$, Germany). After active layer deposition, a bilayer of $5 \mathrm{~nm}$ calcium and $100 \mathrm{~nm}$ aluminum was evaporated through a shadow mask. Solar cells were characterized with a solar simulator (K.H. Steuernagel Lichttechnik GmbH, Germany) employing a 575 W metal halide lamp combined with a filter system to create a spectrum according to AM1.5G conditions, however, with a lower intensity of $70 \mathrm{~mW} \mathrm{~cm}^{-2}$. Current-voltage curves were taken with a Keithley 236 Source Measure Unit (SMU) in a glovebox.

Steady-State Spectroscopy. Steady state absorption spectra were measured with a Perkin Elmer Lambda 25 spectrometer. The layer thickness was determined with a Tencor P10 surface profilometer.

Transient absorption spectroscopy. Transient absorption (TA) measurements were performed with a home-built pump-probe setup. ${ }^{26-27}$ To measure in the time range of 1-4 ns with a resolution of $\sim 100 \mathrm{fs}$, the output of a commercial titanium:sapphire amplifier (Coherent LIBRA-HE, $3.5 \mathrm{~mJ}, 1 \mathrm{kHz}, 100 \mathrm{fs}$ ) was split into two beams that pumped two independent commercial optical parametric amplifiers (Coherent OPerA Solo). One optical parametric 
amplifier (OPA) was used to generate the tunable excitation pulses in the visible, while the second OPA was used to generate the pump beam for white-light generation. For TA measurements in the NIR spectral range covering $1100-2000 \mathrm{~nm}$ a $2100 \mathrm{~nm}$ pump was used to generate white-light in an yttrium vanadate window. Furthermore, a dichroic mirror was used to separate the residual seed beam (idler of the OPA at $2100 \mathrm{~nm}$ ) from the broadband NIR supercontinuum. The variable delay of up to 4 ns between pump and probe was introduced by a broadband retroreflector mounted on a mechanical delay stage. Mostly reflective elements were used to guide the probe beam to the sample to minimize chirp. The excitation pulse was chopped at $500 \mathrm{~Hz}$, while the white-light pulses were dispersed onto a Peltier-cooled 512 pixel long linear extended InGaAs array (Entwicklungsbüro Stresing) which was read out at $1 \mathrm{kHz}$ by home-built electronics. Adjacent diode readings corresponding to the transmission of the sample after an excitation pulse and without an excitation pulse were used to calculate $\Delta \mathrm{T} / \mathrm{T}$.

For measurements in the time range between $1 \mathrm{~ns}$ to $1 \mathrm{~ms}$ with a resolution of $600 \mathrm{ps}$, the excitation pulse was provided by an actively Q-switched $\mathrm{Nd}: \mathrm{YVO}_{4}$ laser (AOT Ltd. MOPA) at $532 \mathrm{~nm}$. In this case the delay between pump and probe was controlled by an electronic delay generator (Stanford Research Systems DG535). TA measurements were performed at room temperature under a dynamic vacuum of $<10^{-5}$ mbar.

Multi-variate curve resolution. MCR analysis is a soft-modelling approach used to factor experimentally measured TA data surfaces into their component spectra and respective concentration profiles applying certain physical constraints such as non-negativity of excited state concentrations or non-positivity of spectra. The MCR analysis and application to TA data has recently been reported and reviewed by us in a separate publication. ${ }^{19-20}$ 


\section{ASSOCIATED CONTENT}

Supporting Information. J-V curves of photovoltaic devices, additional TA spectra and dynamics of pristine and triplet sensitizer-doped polymer films, absorption spectra oxidized polymer films can be found in the supporting information. This material is available free of charge via the Internet at http://pubs.acs.org.

\section{AUTHOR INFORMATION}

\section{Corresponding Author}

*Email: laquai@mpip-mainz.mpg.de

\section{Notes}

The authors declare no competing financial interests.

\section{ACKNOWLEDGMENT}

D.W.G. acknowledges a Kekulé scholarship of the Fonds der Chemischen Industrie (FCI). I.A.H. thanks the Alexander von Humboldt Foundation, Carl-Zeiss-Stiftung and Max Planck Society for postdoctoral fellowships. F.L. acknowledges the Max Planck Society for funding the Max Planck Research Group.

\section{REFERENCES}

(1) Congreve, D. N.; Lee, J.; Thompson, N. J.; Hontz, E.; Yost, S. R.; Reusswig, P. D.; Bahlke, M. E.; Reineke, S.; Van Voorhis, T.; Baldo, M. A., External Quantum Efficiency Above 100\% in a Singlet-Exciton-Fission-Based Organic Photovoltaic Cell. Science 2013, 340, 334-337. 
(2) Chan, W.-L.; Ligges, M.; Jailaubekov, A.; Kaake, L.; Miaja-Avila, L.; Zhu, X.-Y., Observing the Multiexciton State in Singlet Fission and Ensuing Ultrafast Multielectron Transfer. Science 2011, 334, 1541-1545.

(3) Rao, A.; Chow, P. C. Y.; Gelinas, S.; Schlenker, C. W.; Li, C.-Z.; Yip, H.-L.; Jen, A. K. Y.; Ginger, D. S.; Friend, R. H., The Role of Spin in the Kinetic Control of Recombination in Organic Photovoltaics. Nature 2013, 500, 435-439.

(4) Dimitrov, S. D.; Wheeler, S.; Niedzialek, D.; Schroeder, B. C.; Utzat, H.; Frost, J. M.; Yao, J.; Gillett, A.; Tuladhar, P. S.; McCulloch, I., et al., Polaron Pair Mediated Triplet Generation in Polymer/Fullerene Blends. Nat. Commun. 2015, 6, 6501.

(5) Chow, P. C. Y.; Gélinas, S.; Rao, A.; Friend, R. H., Quantitative Bimolecular Recombination in Organic Photovoltaics through Triplet Exciton Formation. J. Am. Chem. Soc. 2014, 136, 3424-3429.

(6) Westenhoff, S.; Howard, I. A.; Hodgkiss, J. M.; Kirov, K. R.; Bronstein, H. A.; Williams, C. K.; Greenham, N. C.; Friend, R. H., Charge Recombination in Organic Photovoltaic Devices with High Open-Circuit Voltages. J. Am. Chem. Soc. 2008, 130, 13653-13658.

(7) Di Nuzzo, D.; Aguirre, A.; Shahid, M.; Gevaerts, V. S.; Meskers, S. C. J.; Janssen, R. A. J., Improved Film Morphology Reduces Charge Carrier Recombination into the Triplet Excited State in a Small Bandgap Polymer-Fullerene Photovoltaic Cell. Adv. Mater. 2010, 22, 43214324. 
(8) Basel, T.; Huynh, U.; Zheng, T.; Xu, T.; Yu, L.; Vardeny, Z. V., Optical, Electrical, and Magnetic Studies of Organic Solar Cells Based on Low Bandgap Copolymer with Spin 1/2 Radical Additives. Adv. Funct. Mater. 2015, 25, 1895-1902.

(9) Etzold, F.; Howard, I. A.; Forler, N.; Melnyk, A.; Andrienko, D.; Hansen, M. R.; Laquai, F., Sub-ns Triplet State Formation by Non-Geminate Recombination in PSBTBT:PC70BM and PCPDTBT:PC60BM Organic Solar Cells. Energy Environ. Sci. 2015, 8, 1511-1522.

(10) Liang, Y.; Xu, Z.; Xia, J.; Tsai, S.-T.; Wu, Y.; Li, G.; Ray, C.; Yu, L., For the Bright Future-Bulk Heterojunction Polymer Solar Cells with Power Conversion Efficiency of 7.4\%. Adv. Mater. 2010, 22, E135-E138.

(11) Chen, H.-Y.; Hou, J.; Zhang, S.; Liang, Y.; Yang, G.; Yang, Y.; Yu, L.; Wu, Y.; Li, G., Polymer Solar Cells with Enhanced Open-Circuit Voltage and Efficiency. Nat. Photon. 2009, 3, 649-653.

(12) Liang, Y.; Feng, D.; Wu, Y.; Tsai, S.-T.; Li, G.; Ray, C.; Yu, L., Highly Efficient Solar Cell Polymers Developed via Fine-Tuning of Structural and Electronic Properties. J. Am. Chem. Soc. 2009, 131, 7792-7799.

(13) Guo, J.; Liang, Y.; Szarko, J.; Lee, B.; Son, H. J.; Rolczynski, B. S.; Yu, L.; Chen, L. X., Structure, Dynamics, and Power Conversion Efficiency Correlations in a New Low Bandgap Polymer: PCBM Solar Cell. J. Phys. Chem. B 2010, 114, 742-748.

(14) Rolczynski, B. S.; Szarko, J. M.; Son, H. J.; Liang, Y.; Yu, L.; Chen, L. X., Ultrafast Intramolecular Exciton Splitting Dynamics in Isolated Low-Band-Gap Polymers and Their Implications in Photovoltaic Materials Design. J. Am. Chem. Soc. 2012, 134, 4142-4152. 
(15) Rolczynski, B. S.; Szarko, J. M.; Son, H. J.; Yu, L.; Chen, L. X., Effects of Exciton Polarity in Charge-Transfer Polymer/PCBM Bulk Heterojunction Films. J. Phys. Chem. Lett. 2014, 5, 1856-1863.

(16) Carsten, B.; Szarko, J. M.; Son, H. J.; Wang, W.; Lu, L.; He, F.; Rolczynski, B. S.; Lou, S. J.; Chen, L. X.; Yu, L., Examining the Effect of the Dipole Moment on Charge Separation in Donor-Acceptor Polymers for Organic Photovoltaic Applications. J. Am. Chem. Soc. 2011, 133, 20468-20475.

(17) Szarko, J. M.; Guo, J.; Rolczynski, B. S.; Chen, L. X., Current Trends in the Optimization of Low Band Gap Polymers in Bulk Heterojunction Photovoltaic Devices. J. Mater. Chem. 2011, $21,7849-7857$.

(18) Szarko, J. M.; Rolczynski, B. S.; Lou, S. J.; Xu, T.; Strzalka, J.; Marks, T. J.; Yu, L.; Chen, L. X., Photovoltaic Function and Exciton/Charge Transfer Dynamics in a Highly Efficient Semiconducting Copolymer. Adv. Funct. Mater. 2014, 24, 10-26.

(19) Jaumot, J.; Gargallo, R.; de Juan, A., A Graphical User-Friendly Interface for MCR-ALS: a New Tool for Multivariate Curve Resolution in MATLAB. Chemom. Intell. Lab. Syst. 2005, 76, 101-110.

(20) Howard, I. A.; Mangold, H.; Etzold, F.; Gehrig, D.; Laquai, F., Ultrafast Dynamics in Molecules, Nanostructures and Interfaces 2014, Volume 8, World Scientific.

(21) Gehrig, D. W.; Roland, S.; Howard, I. A.; Kamm, V.; Mangold, H.; Neher, D.; Laquai, F., Efficiency-Limiting Processes in Low-Bandgap Polymer:Perylene Diimide Photovoltaic Blends. J. Phys. Chem. C. 2014, 118, 20077-20085. 
(22) Sharenko, A.; Gehrig, D.; Laquai, F.; Nguyen, T.-Q., The Effect of Solvent Additive on the Charge Generation and Photovoltaic Performance of a Solution-Processed Small Molecule:Perylene Diimide Bulk Heterojunction Solar Cell. Chem. Mater. 2014, 26, 4109-4118.

(23) Koster, L. J. A.; Mihailetchi, V. D.; Blom, P. W. M., Bimolecular Recombination in Polymer/Fullerene Bulk Heterojunction Solar Cells. Appl. Phys. Lett. 2006, 88, 052104.

(24) Burke, T. M.; McGehee, M. D., How High Local Charge Carrier Mobility and an Energy Cascade in a Three-Phase Bulk Heterojunction Enable $>90 \%$ Quantum Efficiency. Adv. Mater. 2014, 26, 1923-1928.

(25) Vukmirović, N.; Ponseca, C. S.; Němec, H.; Yartsev, A.; Sundström, V., Insights into the Charge Carrier Terahertz Mobility in Polyfluorenes from Large-Scale Atomistic Simulations and Time-Resolved Terahertz Spectroscopy. J. Phys. Chem. C. 2012, 116, 19665-19672.

(26) Etzold, F.; Howard, I. A.; Mauer, R.; Meister, M.; Kim, T.-D.; Lee, K.-S.; Baek, N. S.; Laquai, F., Ultrafast Exciton Dissociation Followed by Nongeminate Charge Recombination in PCDTBT:PCBM Photovoltaic Blends. J. Am. Chem. Soc. 2011, 133, 9469-9479.

(27) Gehrig, D.; Howard, I. A.; Kamm, V.; Dyer-Smith, C.; Etzold, F.; Laquai, F., Charge Generation in Polymer:Perylene Diimide Blends Probed by Vis-NIR Broadband Transient Absorption Pump-Probe Spectroscopy. Poc. SPIE 2013, 8811, 88111F-88111F-10. 


\section{TOC GRAPHICS}

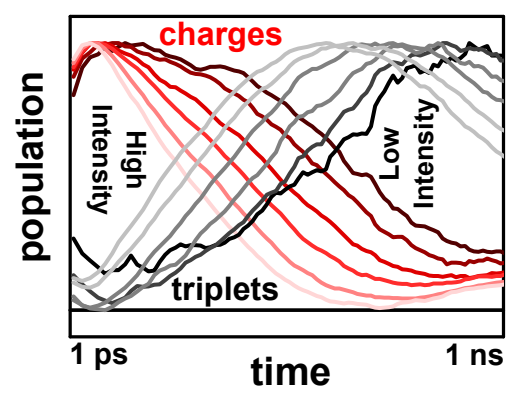

For Table of Contents Only 